\title{
Analytic Comparison of MHD Squeezing Flow in Porous Medium with Slip Condition
}

\author{
Inayat Ullah, M. T. Rahim, Hamid Khan, and Mubashir Qayyum \\ Department of Mathematics, National University of Computer and Emerging Sciences, FAST, Peshawar Campus, Pakistan \\ Correspondence should be addressed to Inayat Ullah; p119952@nu.edu.pk
}

Received 10 October 2015; Accepted 11 January 2016

Academic Editor: Ashok Chatterjee

Copyright (C) 2016 Inayat Ullah et al. This is an open access article distributed under the Creative Commons Attribution License, which permits unrestricted use, distribution, and reproduction in any medium, provided the original work is properly cited.

\begin{abstract}
The aim of this paper is to compare the efficiency of various techniques for squeezing flow of an incompressible viscous fluid in a porous medium under the influence of a uniform magnetic field squeezed between two large parallel plates having slip boundary. Fourth-order nonlinear ordinary differential equation is obtained by transforming the Navier-Stokes equations. Resulting boundary value problem is solved using Differential Transform Method (DTM), Daftardar Jafari Method (DJM), Adomian Decomposition Method (ADM), Homotopy Perturbation Method (HPM), and Optimal Homotopy Asymptotic Method (OHAM). The problem is also solved numerically using Mathematica solver NDSolve. The residuals of the problem are used to compare and analyze the efficiency and consistency of the abovementioned schemes.
\end{abstract}

\section{Introduction}

The study of squeezing flow started in 19th century and it continues to receive considerable attention due to its practical applications in physical and biophysical areas, namely, food industry, chemical engineering, polymer processing, compression, and injection modeling. Stefan [1] accomplished elementary research in this field. Analysis of Newtonian fluid squeezed between two infinite planar plates is studied by Ran et al. [2]. Thin Newtonian liquid films squeezing between two plates were studied by Grimm [3]. Squeezing flow under the influence of magnetic field is broadly applied to bearing with liquid-metal lubrication [4-7].

The study of magnetic field effects on lubrication fluid films has attracted many scientists for a number of years. The flows of electrically conducting fluid through porous medium have attained incomparable status and have been the limelight of concern of many researchers in the last few decades. The particular applications are investigated in the study of ground water flow, irrigation problems, crude petroleum recovery, heat-storage beds, thermal and insulating engineering, chromatography, chemical catalytic reactors, and many more. Hughes and Elco [8] investigated the dynamics of an electrically conducting fluid in the presence of magnetic field between two parallel disks, one rotating at a constant angular velocity, for two cases, an axial magnetic field with a radial current and a radial magnetic field with an axial current. They discovered that the magnetic field affects the load capacity of the bearing and that the frictional torque on the rotor becomes zero for both the cases by applying electrical energy through the electrodes to the fluid. Ullah et al. studied the squeezing flow, in a porous medium, of a Newtonian fluid under the influence of imposed magnetic field [9]. The velocity profile of the fluid is discussed in the last work by considering various relations between the values of Reynolds and Hartmann number.

High order nonlinear boundary value problems arise in the study of squeezing flow of Newtonian as well as nonNewtonian fluids. The exact solution of these problems is sometimes difficult to find due to the mathematical complexity of Navier-Stokes equations. In order to solve these problems, various seminumerical techniques are widely used. We discuss here one by one these techniques and apply them to obtain the velocity profile of the fluid.

Homotopy Perturbation Method (HPM) was first introduced by He [10, 11]. Marinca et al. [12, 13] introduced OHAM for approximate solution of nonlinear problems of thin film flow of a fourth-grade fluid down a vertical cylinder and for 
the study of the behavior of nonlinear mechanical vibration of electrical machines. It is scrutinized that HPM and HAM are the special cases of OHAM [14].

Differential Transform Method (DTM) was initially introduced by Zhou in 1986 [15]. Islam et al. [16] successfully applied this technique for squeezing flow of a Newtonian fluid in porous medium channel. Ullah et al. [17] investigated the squeezing fluid flow under the influence of magnetic field with slip boundary condition using DTM. Ayaz [18] studied the applications of two-dimensional DTM in case of partial differential equations. Hassan [19] compared DTM with ADM in solving PDEs.

Adomian [20, 21] (1923-1996), in 1980, introduced Adomian Decomposition Method for solving nonlinear functional equations. The technique is based on the decomposition of solution of nonlinear operator equation in a series of functions. Wazwaz [22] introduced the modified form of ADM and used it in many BVPs successfully. The basic idea of Daftardar Jafari Method (DJM) is introduced by Daftardar-Gejji et al. [23, 24] to solve fractional boundary value problems with Dirichlet boundary conditions. The solution of fifth- and sixth-order boundary value problem using DJM is studied by Ullah et al. and they got excellent results [25].

The goal of this research paper is to solve the model of squeezing flow of a Newtonian fluid in a porous medium with MHD effect by using HPM, OHAM, DTM, ADM, NIM, and the Mathematica solver NDSolve. Furthermore, to check the efficiency of each scheme, the residuals of the problem are used. Preparation of the model and basic ideas of the mentioned techniques along with their applications are discussed in the respective sections.

\section{Problem Modeling}

The continuity and momentum equation for steady squeezing flow in a porous medium under the influence of magnetic field, as shown in Figure 1, are

$$
\begin{aligned}
& \nabla \cdot W=0, \\
& \rho D W=\nabla \cdot T+J \times B+r .
\end{aligned}
$$

Here $W$ is the velocity vector, $\nabla$ is the material time derivative, and $T$ is the Cauchy stress tensor given by $T=-p I+\mu A$ with $A=\nabla W+(\nabla W)^{t} . B$ is the total magnetic field given by $B=B_{0}+b . B_{0}$ and $b$ represent the imposed and induced magnetic fields, respectively. $r$ is Darcy's resistance given by $[26,27]$

$$
r=-\frac{\mu W}{k}
$$

The magnetohydrodynamic force can be written as follows:

$$
J \times B=-\sigma B_{0}^{2} W
$$

Suppose that the magnetic field is applied along $z$-axis and the plates are nonconducting. For small velocity $w$, the gap distance $2 L$ between the plates changes slowly with time $t$ so that it can be taken constant. The flow is axisymmetric with

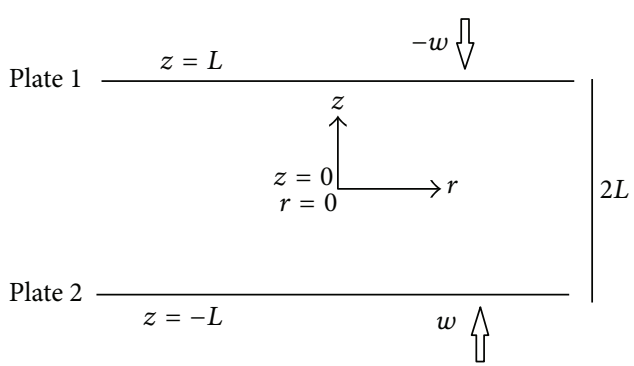

Figure 1: Geometry of the squeezing flow.

$z$-axis perpendicular to plates and $z= \pm L$ at the plates. The components of $W$ for the present case are $W=\left(w_{r}, 0, w_{z}\right)$. If $P=(\rho / 2)\left(w_{r}^{2}+w_{z}^{2}\right)+p$ is the generalized pressure and the flow is steady then by comparing components the Navier-Stokes equations (1) can be written as

$$
\begin{aligned}
\frac{\partial P}{\partial r} & -\rho\left(\frac{\partial w_{z}}{\partial r}-\frac{\partial w_{r}}{\partial z}\right) w_{z} \\
& =-\left(\mu \frac{\partial}{\partial z}\left(\frac{\partial w_{z}}{\partial r}-\frac{\partial w_{r}}{\partial z}\right)+\left(\frac{\mu}{k}+\sigma B_{0}^{2}\right) w_{r}\right), \\
\frac{\partial P}{\partial z} & +\rho\left(\frac{\partial w_{z}}{\partial r}-\frac{\partial w_{r}}{\partial z}\right) w_{r} \\
& =\frac{\mu}{r} \frac{\partial}{\partial r}\left(r\left(\frac{\partial w_{z}}{\partial r}-\frac{\partial w_{r}}{\partial z}\right)\right)-\left(\frac{\mu}{k}\right) w_{z} .
\end{aligned}
$$

Introducing stream function $\psi(r, z)$ [9], eliminating the generalized pressure $P$ from (4) and (5), and using the transformation $\psi(r, z)=r^{2} t(z)$ and the boundary conditions

$$
\begin{gathered}
\text { at } z=0 \\
w_{z}=0, \\
\frac{\partial w_{r}}{\partial z}=0, \\
\text { at } z=L \quad \\
w_{r}=\beta \frac{\partial w_{r}}{\partial z}, \\
w_{z}=-w .
\end{gathered}
$$

We have

$$
t^{(\mathrm{iv})}(z)-\left(\frac{1}{k}+\frac{\sigma B_{0}^{2}}{\mu}\right) t^{\prime \prime}(z)+2 \frac{\rho}{\mu} t(z) t^{\prime \prime \prime}(z)=0,
$$

subject to boundary conditions

$$
\begin{aligned}
t(0) & =0, \\
t^{\prime \prime}(0) & =0, \\
t(L) & =\frac{w}{2}, \\
t^{\prime}(L) & =\beta t^{\prime \prime}(L) .
\end{aligned}
$$


Introducing nondimensional parameters,

$$
\begin{aligned}
& T^{*}=\frac{t}{w / 2}, \\
& z^{*}=\frac{z}{L}, \\
& \mathscr{R}=\frac{\rho L w}{\mu}, \\
& \mathscr{M}=L \sqrt{\frac{1}{k}+\frac{\sigma B_{0}^{2}}{\mu}} .
\end{aligned}
$$

Omitting ${ }^{*},(8)$ and (9) become

$$
\begin{aligned}
T^{(\mathrm{iv})}(z)-\mathscr{M}^{2} T^{\prime \prime}(z)+\mathscr{R} T(z) T^{\prime \prime \prime}(z) & =0, \\
T(0) & =0, \\
T^{\prime \prime}(0) & =0, \\
T(1) & =1, \\
T^{\prime}(1) & =\gamma T^{\prime \prime}(1) .
\end{aligned}
$$

We solve (11) and (12) by fixing $\mathscr{M}=1, \mathscr{R}=1$, and $\gamma=1$ to find the particular solution in each case for comparison purpose.

\section{Basic Idea and Application of DTM}

For the function $t(z)$, one-dimensional differential transform is defined as follows $[28,29]$ :

$$
T(z)=\frac{1}{k !}\left[\frac{d^{k} t(z)}{d z^{k}}\right]_{z=0} .
$$

The inverse transform of $T(z)$ is defined as follows:

$$
t(z)=\sum_{k=0}^{\infty} z^{k} T(z) .
$$

Combining (13) and (14), we can write

$$
t(z)=\sum_{k=0}^{\infty} \frac{z^{k}}{k !}\left[\frac{d^{k} t(z)}{d z^{k}}\right]
$$

$t(z)$ in a finite series is as follows:

$$
t(z)=\sum_{k=0}^{N} \frac{z^{k}}{k !}\left[\frac{d^{k} t(z)}{d z^{k}}\right],
$$

which means that

$$
t(z)=\sum_{k=N+1}^{\infty} \frac{z^{k}}{k !}\left[\frac{d^{k} t(z)}{d z^{k}}\right]
$$

can be considered negligibly small.

Some fundamental theorems on one-dimensional differential transform are as follows.
Theorem 1. If $t(z)=f(z) \pm h(z)$, then $T(k)=F(k) \pm H(k)$.

Theorem 2. If $t(z)=h^{(n)}(z)$, then $T(z)=((k+n) ! / k !) H(k+$ $n)$.

Theorem 3. Ift $(z)=h(z) \cdot f(z)$, then $T(z)=\sum_{r=0}^{k} H(r) F(k-$ $r)$.

Theorem 4. If $t(z)=z^{n}$, then

$$
T(z)=\delta(k-n)= \begin{cases}1 & \text { if } k=n \\ 0 & \text { if } k \neq n\end{cases}
$$

Keeping in view the abovementioned theorems, the differential transform of (11) is given by

$$
\begin{gathered}
\widetilde{T}(n+4)=\frac{n !}{(n+4) !}\left(m^{2}(n+1)(n+2) \widetilde{T}(n+2)\right. \\
\left.-R \sum_{r=0}^{n}(r+1)(r+2)(r+3) \widetilde{T}(r+3) \widetilde{T}(n-r)\right),
\end{gathered}
$$

with transformed boundary conditions

$$
\begin{aligned}
& \widetilde{T}(0)=0, \\
& \widetilde{T}(1)=\alpha, \\
& \widetilde{T}(2)=0, \\
& \widetilde{T}(3)=\beta .
\end{aligned}
$$

Using (19) and (20), the values of $\widetilde{T}(i), i=1,2,3, \ldots, 15$, are

$$
\begin{aligned}
& \widetilde{T}(2 n)=0, \quad \text { for } n=0(1) 7 \\
& \widetilde{T}(5)=\frac{1}{120}(6 \beta-6 \alpha \beta) \\
& \widetilde{T}(7)=\frac{1}{840 \beta}\left(1-4 \alpha+3\left(-2 \beta+\alpha^{2}\right)\right),
\end{aligned}
$$

$$
\begin{aligned}
& \widetilde{T}(9)=\frac{\beta}{60480}\left(1-9 \alpha+3 \alpha\left(32 \beta-5 \alpha^{2}\right)+(-72 \beta\right. \\
& \left.\left.+23 \alpha^{2}\right)\right),
\end{aligned}
$$

$$
\begin{aligned}
& \widetilde{T}(11)=\frac{\beta}{6652800}\left(1-16 \alpha-44 \alpha\left(-39 \beta+4 \alpha^{2}\right)\right. \\
& \left.+\left(-414 \beta+86 \alpha^{2}\right)+3\left(432 \beta^{2}-482 \alpha^{2} \beta+35 \alpha^{4}\right)\right),
\end{aligned}
$$

$$
\begin{aligned}
& \widetilde{T}(13)=\frac{\beta}{1037836800}(1-25 \alpha+2 \alpha(7446 \beta \\
& \left.-475 \alpha^{2}\right)+2\left(-948 b+115 \alpha^{2}\right)-9 \alpha\left(7384 \beta^{2}\right. \\
& \left.-2620 \alpha^{2} \beta+105 \alpha^{4}\right)+3\left(14616 \beta^{2}-11808 \alpha^{2} \beta\right. \\
& \left.\left.+563 \alpha^{4}\right)\right),
\end{aligned}
$$




$$
\begin{aligned}
& \widetilde{T}(15)=\frac{\beta}{217945728000}(1-36 \alpha+24 \alpha(4106 \beta \\
& \left.-145 \alpha^{2}\right)+\left(-7974 \beta+505 \alpha^{2}\right)-12 \alpha\left(239508 \beta^{2}\right. \\
& \left.-62828 \alpha^{2} \beta+1627 \alpha^{4}\right)+\left(703296 \beta^{2}\right. \\
& \left.-427716 \alpha^{2} \beta+12139 \alpha^{4}\right)+9\left(-151344 \beta^{3}\right. \\
& \left.\left.+282256 \alpha^{2} \beta^{2}-47590 \alpha^{4} \beta+1155 \alpha^{6}\right)\right) .
\end{aligned}
$$

To find the values of $\alpha$ and $\beta$, we use the following transformed boundary conditions:

$$
\begin{array}{r}
\sum_{n=0}^{15}(n \widetilde{T}[n]-n(n-1) \widetilde{T}[n])=0, \\
\sum_{n=0}^{15} \widetilde{T}[n]=1,
\end{array}
$$

which leads us to the following values:

$$
\begin{aligned}
& \alpha=0.754966, \\
& \beta=0.242565 .
\end{aligned}
$$

The approximate solution of the problem is as follows:

$$
\begin{aligned}
T(z)= & 0.754966 z+0.242565 z^{3}+0.00297182 z^{5} \\
& -0.00050977 z^{7}+3.913152541684915 \\
& \times 10^{-6} z^{9}+3.1529964918115006 \times 10^{-6} z^{11} \\
& -1.2149332568415323 \times 10^{-7} z^{13} \\
& -2.0892317176411676 \times 10^{-8} z^{15}
\end{aligned}
$$

\section{Basic Idea and Application of DJM}

Consider the nonlinear boundary value problem $[9,23,24]$ :

$$
\mathfrak{Q}(T(z))+\mathfrak{M}(T(z))+\mathfrak{N}(T(z))=f(z),
$$

where $\mathfrak{Q}$ represents the highest order derivative with respect to $z, \mathfrak{M}$ is the linear term reminder, and $\mathfrak{N}$ represents the nonlinear term. Using the operator $\mathbb{L}=d^{4} / d x^{4},(25)$ becomes

$$
\begin{aligned}
T(z)= & \eta_{0}+\eta_{1} z+\eta_{2} \frac{z^{2}}{2 !}+\eta_{3} \frac{z^{3}}{3 !}+\mathfrak{Q}^{-1} f(z) \\
& -\mathfrak{Q}^{-1} \mathfrak{M}(T(z))-\mathfrak{Q}^{-1} \mathfrak{N}(T(z)) .
\end{aligned}
$$

$\eta_{i}$ are constants to be determined later. The function $T(z)$ is then expressed by the infinite series as

$$
T(z)=\sum_{k=0}^{\infty} T_{k}(z) .
$$

The nonlinear term $\mathfrak{N}(T(z))$ is written in the sum of Daftardar-Geiji et al. polynomials as

$$
\mathfrak{N}(T(z))=\sum_{n=0}^{\infty} G_{n} .
$$

Here $G_{n}^{\prime}$ s are defined as

$$
\begin{aligned}
G_{0}(z) & =\mathfrak{N}\left(T_{0}(z)\right), \\
G_{m}(z) & =\mathfrak{N}\left(\sum_{n=0}^{m} T_{n}(z)\right)-\mathfrak{N}\left(\sum_{n=0}^{m-1} T_{n}(z)\right) .
\end{aligned}
$$

Using these $G_{n}^{\prime}$ s, we have the following components of $T(z)$ :

$$
\begin{aligned}
T_{0}(z) & =\beta_{0}+\beta_{1} z+\beta_{2} \frac{z^{2}}{2 !}+\beta_{3} \frac{z^{3}}{3 !}-L^{-1} f(z), \\
T_{k+1}(z) & =-L^{-1} \mathscr{M}\left(T_{k}(z)\right)-L^{-1}\left(G_{k}\right) .
\end{aligned}
$$

For the solution of (11) with the help of (12), we have $f(z)=$ $0, \mathfrak{M}(T(z))=-m^{2} T^{\prime \prime}(z)$, and $\mathfrak{N}(T(z))=R T(z) T^{\prime \prime \prime}(z)$ the components of $T(z)$ using DJM are as follows:

$$
\begin{aligned}
T_{0}(z)= & B z+\frac{A z^{3}}{6}, \\
T_{1}(z)= & \frac{1}{120}(A-A B) z^{5}-\frac{A^{2} z^{7}}{5040}, \\
T_{2}(z)= & \frac{\left(A-4 A B+3 A B^{2}\right) z^{7}}{5040} \\
& +\left(-\frac{A^{2}}{30240}+\frac{A^{2} B}{22680}\right) z^{9} \\
& +\frac{\left(-7 A^{2}+12 A^{3}+14 A^{2} B-7 A^{2} B^{2}\right) z^{11}}{13305600} \\
& +\frac{\left(A^{3}-A^{3} B\right) z^{13}}{38438400}-\frac{A^{4} z^{15}}{3962649600} .
\end{aligned}
$$

$T_{3}(z)$ is also obtained in the same manner. The series solution up to $T_{3}(z)$ is then given by 


$$
\begin{aligned}
T(z)= & T_{0}(z)+T_{1}(z)+T_{2}(z)+T_{3}(z)+O\left(z^{14}\right) \\
= & B z+\frac{A z^{3}}{6}+\frac{1}{120}(A-A B) z^{5}+\left(-\frac{A^{2}}{5040}+\frac{A-4 A B+3 A B^{2}}{5040}\right) z^{7} \\
& +\left(-\frac{A^{2}}{30240}+\frac{A^{2} B}{22680}+\frac{A-9 A B+23 A B^{2}-15 A B^{3}}{362880}\right) z^{9} \\
& +\left(\frac{-12 A^{2}+61 A^{2} B-55 A^{2} B^{2}}{9979200}+\frac{-7 A^{2}+12 A^{3}+14 A^{2} B-7 A^{2} B^{2}}{13305600}\right) z^{11} \\
& +\left(\frac{A^{3}-A^{3} B}{38438400}+\frac{-183 A^{2}+1056 A^{3}+1041 A^{2} B-1684 A^{3} B-1533 A^{2} B^{2}+675 A^{2} B^{3}}{6227020800}\right) z^{13} .
\end{aligned}
$$

Use the boundary conditions at $z=1$ to get

$$
\begin{aligned}
& A=1.45535, \\
& B=0.754972
\end{aligned}
$$

so that the last equation becomes

$$
\begin{aligned}
T(z)= & 0.75 z+0.24 z^{3}+2.97 \times 10^{-3} z^{5}-5.10 \\
& \times 10^{-4} z^{7}+3.91 \times 10^{-6} z^{9}+3.30 \times 10^{-6} z^{11} \\
& -8.03 \times 10^{-8} z^{13}+O\left(z^{14}\right)
\end{aligned}
$$

\section{Basic Idea and Application of ADM}

Consider the differential equation

$$
\mathfrak{Q}(T(z))+\mathfrak{M}(T(z))+\mathfrak{N}(T(z))=f(z) .
$$

Following the basic concept of DJM, for the nonlinear term $\mathfrak{N}(T(z))$ Adomian introduced polynomials so called Adomian polynomials defined as

$$
A_{n}=\sum_{r=1}^{n} c(r, n) T^{r}\left(z_{0}\right),
$$

where $c(r, n)$ are products (or sum of products) of $r$ components of $T(z)$ whose subscripts sum to $n$, divided by the factorial of the number of repeated subscripts. $\mathfrak{N}(T(z))$ is written in the form of infinite series of Adomian polynomials as

$$
\mathfrak{N}(T(z))=\sum_{k=0}^{\infty} A_{k}
$$

In our case $\mathfrak{N}(T(z))=T(z) T^{\prime \prime \prime}(z)$. Some polynomials for this nonlinear term are

$$
\begin{aligned}
A_{0} & =\mathfrak{N}\left(T_{0}(z)\right)=T_{0}(z) \cdot T_{0}^{\prime \prime \prime}(z), \\
A_{1} & =T_{1}(z) \mathfrak{N}^{\prime}\left(T_{0}(z)\right)=T_{1}(z)\left(T_{0}(z) T_{0}^{(\mathrm{iv})}\right. \\
& \left.+T_{0}^{\prime} T_{0}^{\prime \prime \prime}(z)\right),
\end{aligned}
$$

$$
\begin{aligned}
A_{2} & =T_{2}(z) \mathfrak{N}^{\prime}\left(T_{0}(z)\right)+\frac{T_{1}(z)}{2 !} \mathfrak{N}^{\prime \prime}\left(T_{0}(z)\right)=T_{2}(z) \\
& \cdot\left(T_{0}(z) T_{0}^{(\mathrm{iv})}+T_{0}^{\prime} T_{0}^{\prime \prime \prime}(z)\right) \\
& +\frac{T_{1}(z)}{2 !}\left(T_{0}(z) T_{0}^{(\mathrm{v})}(z)+2 T_{0}^{\prime}(z) T_{0}^{(\mathrm{iv})}(z)\right. \\
& \left.+T_{0}^{\prime \prime \prime}(z) T_{0}^{\prime \prime}(z)\right) .
\end{aligned}
$$

The recursive process to find the components of $T(z)$ is

$$
\begin{gathered}
T_{0}(z)=\beta_{0}+\beta_{1} z+\beta_{2} \frac{z^{2}}{2 !}+\beta_{3} \frac{z^{3}}{3 !}-L^{-1} f(z), \\
T_{k+1}(z)=-L^{-1} M\left(T_{k}(z)\right)-L^{-1}\left(A_{k}\right), \\
k=0,1,2, \ldots .
\end{gathered}
$$

By means of boundary conditions at $z=0$ the components of $T(z)$ are obtained as follows:

$$
\begin{aligned}
T_{0}(z)= & b z+\frac{a z^{3}}{6}, \\
T_{1}(z)= & \frac{1}{120}(a-a b) z^{5}-\frac{a^{2} z^{7}}{5040}, \\
T_{2}(z)= & \frac{(a-a b) z^{7}}{5040}+\frac{\left(-a^{2}-a^{2} b+a^{2} b^{2}\right) z^{9}}{362880} \\
& +\left(-\frac{a^{3}}{1900800}+\frac{a^{3} b}{1814400}\right) z^{11} \\
& +\frac{a^{4} z^{13}}{172972800} .
\end{aligned}
$$


Considering the components up to $T_{4}(z)$, we have the following solution:

$$
\begin{aligned}
& T(z)=b z+\frac{a z^{3}}{6}+\frac{1}{120}(a-a b) z^{5}+\left(-\frac{a^{2}}{5040}+\frac{a-a b}{5040}\right) \\
& \cdot z^{7}+\left(\frac{a-a b}{362880}+\frac{-a^{2}-a^{2} b+a^{2} b^{2}}{362880}\right) z^{9}+\left(-\frac{a^{3}}{1900800}\right. \\
& \left.+\frac{a^{3} b}{1814400}+\frac{a-a b}{39916800}+\frac{-a^{2}-2 a^{2} b+2 a^{2} b^{2}}{39916800}\right) z^{11} \\
& +\left(\frac{a^{4}}{172972800}+\frac{-a^{2}-3 a^{2} b+3 a^{2} b^{2}}{6227020800}\right. \\
& \left.+\frac{-57 a^{3}+59 a^{3} b+a^{3} b^{2}-a^{3} b^{3}}{6227020800}\right) z^{13} \\
& +\left(\frac{-1295 a^{4}+2848 a^{4} b-1463 a^{4} b^{2}}{1307674368000}\right. \\
& \left.+\frac{-112 a^{3}+115 a^{3} b+3 a^{3} b^{2}-3 a^{3} b^{3}}{1307674368000}\right) z^{15} \\
& +\left(\frac{a^{5}}{29804544000}-\frac{251 a^{5} b}{7410154752000}\right. \\
& \left.+\frac{-11513 a^{4}+23653 a^{4} b-11974 a^{4} b^{2}-a^{4} b^{3}+a^{4} b^{4}}{355687428096000}\right) \\
& \cdot z^{17}+\left(-\frac{41 a^{6}}{168951528345600}\right. \\
& \left.+\frac{-26697 a^{5}+309950 a^{5} b-441391 a^{5} b^{2}+157724 a^{5} b^{3}}{121645100408832000}\right) \\
& \cdot z^{19}
\end{aligned}
$$

Use the conditions at $z=1$ to get two equations which, on solving, give

$$
\begin{aligned}
& a=1.44861, \\
& b=0.755971 .
\end{aligned}
$$

The approximate solution thus obtained is

$$
\begin{aligned}
T(z)= & 0.755971 z+0.241436 z^{3}+0.002946 z^{5} \\
& -0.000346 z^{7}-5.875514 \times 10^{-6} z^{9} \\
& -3.958095 \times 10^{-7} z^{11}+1.895084 \times 10^{-8} z^{13} \\
& +1.649921 \times 10^{-11} z^{15}+4.480188 \\
& \times 10^{-11} z^{17}-1.009835 \times 10^{-12} z^{19}
\end{aligned}
$$

\section{Basic Idea and Application of HPM}

Let us consider a nonlinear differential equation as follows $[10,11]$ :

$$
\mathfrak{M}(T)=g(z)
$$

The operator $\mathfrak{M}$ is usually divided into two parts, namely, linear $(\mathfrak{L})$ and nonlinear $(\mathfrak{N})$; that is,

$$
\mathfrak{M}=\mathfrak{Q}+\mathfrak{N},
$$

and $g(z)$ is a known analytic function. Equation (44) can be written as

$$
\mathfrak{Q}(T)+\mathfrak{N}(T)-g(z)=0, \quad r \in \Omega,
$$

with boundary conditions

$$
\mathfrak{B}\left(T, \frac{\partial T}{\partial z}\right)=0, \quad z \in \Gamma
$$

where $\Gamma$ is the boundary of the domain $\Omega$. A homotopy $\mathfrak{H}(T(z, p), p): \mathbf{R} \times[0,1] \rightarrow \mathbf{R}$ is constructed which satisfies

$$
\begin{aligned}
\mathfrak{H}(v, p)= & (1-p)\left[\mathfrak{Q}(v)-\mathfrak{Q}\left(T_{0}\right)\right] \\
& +p[\mathfrak{M} v-g(z)] .
\end{aligned}
$$

$p \in[0,1]$ is an embedding parameter and $T_{0}$ is the first approximation satisfying the boundary conditions. Taylor's series expansion of $T(z, p)$ about $p$ is used for the approximate solution of the differential equation as follows:

$$
T(z)=v_{0}(z)+\sum_{r=1}^{\infty} v_{r}(z) p^{r} .
$$

Putting (49) in (48) and equating the coefficients of like powers of $p$ for the present problem, we get the following:

Zeroth-order problem:

$$
\begin{gathered}
T^{(\mathrm{iv})}(z)=0, \\
T_{0}(0)=0, \\
T_{0}^{\prime \prime}(0)=0, \\
T_{0}(1)=1, \\
T_{0}^{\prime}(1)=T_{0}^{\prime \prime}(1) .
\end{gathered}
$$

First-order problem:

$$
\begin{aligned}
T_{1}^{(\mathrm{iv})}(z) & =T_{0}^{\prime \prime}(z)-T_{0}(z) T_{0}^{\prime \prime \prime}(z), \\
T_{1}(0) & =0, \\
T_{1}^{\prime \prime}(0) & =0, \\
T_{1}(1) & =0, \\
T_{1}^{\prime}(1) & =T_{1}^{\prime \prime}(1) .
\end{aligned}
$$

Second-order problem:

$$
\begin{aligned}
T_{2}^{(\mathrm{iv})}(z) & =T_{1}^{\prime \prime}(z)-T_{1}(z) T_{0}^{\prime \prime \prime}(z)-T_{0}(z) T_{1}^{\prime \prime \prime}(z), \\
T_{2}(0) & =0 \\
T_{2}^{\prime \prime}(0) & =0 \\
T_{2}(1) & =0 \\
T_{2}^{\prime}(1) & =T_{2}^{\prime \prime}(1) .
\end{aligned}
$$


Third-order problem:

$$
\begin{aligned}
T_{3}^{(\mathrm{iv})}(z)= & T_{2}^{\prime \prime}(z)-T_{2}(z) T_{0}^{\prime \prime \prime}(z)-T_{1}(z) T_{1}^{\prime \prime \prime}(z) \\
& -T_{0}(z) T_{2}^{\prime \prime \prime}(z) \\
T_{3}(0)= & 0 \\
T_{3}^{\prime \prime}(0)= & 0 \\
T_{3}(1)= & 0 \\
T_{3}^{\prime}(1)= & T_{3}^{\prime \prime}(1) .
\end{aligned}
$$

Fourth-order problem:

$$
\begin{aligned}
T_{4}^{(\mathrm{iv})}(z)= & T_{3}^{\prime \prime}(z)-T_{3}(z) T_{0}^{\prime \prime \prime}(z)-T_{2}(z) T_{1}^{\prime \prime \prime}(z) \\
& -T_{1}(z) T_{2}^{\prime \prime \prime}(z)-T_{0}(z) T_{3}^{\prime \prime \prime}(z), \\
T_{4}(0)= & 0 \\
T_{4}^{\prime \prime}(0)= & 0 \\
T_{4}(1)= & 0 \\
T_{4}^{\prime}(1)= & T_{4}^{\prime \prime}(1) .
\end{aligned}
$$

By considering the fourth-order solution, we have

$$
\begin{aligned}
T(z)= & T_{0}(z)+T_{1}(z)+T_{2}(z)+T_{3}(z)+T_{4}(z) \\
= & 0.755 z+0.243 z^{3}+0.003 z^{5}-5.097 \times 10^{-4} z^{7} \\
& +3.877 \times 10^{-6} z^{9}+3.150 \times 10^{-6} z^{11}-1.293 \\
& \times 10^{-7} z^{13}+O\left(z^{14}\right)
\end{aligned}
$$

\section{Basic Idea and Application of OHAM}

If $t(z)$ is an unknown function, $f(z)$ is known function, and $\mathfrak{R}, \mathfrak{N}, \mathfrak{B}$ are linear, nonlinear, and boundary operator, respectively, then for boundary value problem [12-14]

$$
\mathfrak{Q}[t(z)]+f(z)+\mathfrak{N}[t(z)]=0,
$$

with boundary conditions

$$
\mathfrak{B}\left(T(z), \frac{d T(z)}{d z}\right)=0,
$$

a homotopy $\mathfrak{H}(T(z, p), p): \mathbf{R} \times[0,1] \rightarrow \mathbf{R}$ is constructed which satisfies the following:

$$
\begin{aligned}
& (1-p)[\mathfrak{Q}(T(z, p))+f(z)] \\
& \quad=\mathfrak{H}(p)[\mathfrak{Q}(T(z, p))+f(z)+\mathfrak{N}(T(z, p))], \\
& \mathfrak{B}\left(T(z, p), \frac{\partial T(z, p)}{\partial z}\right)=0,
\end{aligned}
$$

where $p \in[0,1]$ and $\mathfrak{H}(p)$ is a nonzero auxiliary function. If $p=0$, then $T(z, 0)=t_{0}(z)$ and if $p=1$, then $T(z, 1)=t(z)$ hold. It means that the solution $T(z, p)$ approaches from $t_{0}(z)$ to $t(z)$ as $p$ varies from 0 to 1 .

For $p=0$

$$
\begin{aligned}
\mathfrak{L}\left(T_{0}(z)\right)+f(z) & =0, \\
\mathfrak{B}\left(T_{0}, \frac{d T_{0}}{d z}\right) & =0 .
\end{aligned}
$$

The auxiliary function $\mathfrak{S}(p)$ is selected such that

$$
\mathfrak{S}(p)=\sum_{k=0}^{n} p^{k} C_{k}
$$

where $C_{k}$ are the convergence controlling constants to be determined. Expanding $T(z, p)$ in Taylor's series about $p$ to get

$$
T\left(z, p, C_{k}\right)=T_{0}(z)+\sum_{j=1}^{n} T_{j}\left(z, C_{1}, C_{2}, \ldots, C_{j}\right) p^{j},
$$

substituting (61) into (58) and comparing the coefficients of the same powers of $p$, the general $n$th order problem is

$$
\begin{aligned}
\mathfrak{L} & \left(T_{n}(z)\right)-\mathfrak{L}\left(T_{n-1}(z)\right)=C_{n} \mathfrak{N}_{0}\left(T_{0}(z)\right) \\
& +\left(\sum _ { j = 1 } ^ { n - 1 } C _ { j } \left[\mathfrak{L}\left(T_{n-j}(z)\right)\right.\right. \\
& \left.\left.+\mathfrak{N}_{n-j}\left(T_{0}(z), T_{1}(z), \ldots, T_{n-1}(z)\right)\right]\right),
\end{aligned}
$$

with boundary conditions

$$
\mathfrak{B}\left(T_{n}, \frac{d T_{n}}{d z}\right)=0, \quad n=1,2,3,4, \ldots
$$

where $\mathfrak{N}_{m}\left(T_{0}(z), T_{1}(z), \ldots, T_{m-1}(z)\right)$ is the coefficient of $p^{m}$ in the expansion of $\mathfrak{N}(T(z, p))$ about $p$. Consider

$$
\begin{aligned}
\mathfrak{N}\left(T\left(z, p, C_{k}\right)\right)= & \mathfrak{N}_{0}\left(T_{0}(z)\right) \\
& +\sum_{m=1}^{\infty} \mathfrak{N}_{m}\left(T_{0}, T_{1}, T_{2}, \ldots, T_{m}\right) p^{m} .
\end{aligned}
$$

The $k$ th-order approximation $\widetilde{T}$ is

$$
\begin{aligned}
\widetilde{T}\left(z, C_{1}, C_{2}, \ldots, C_{k}\right)= & T_{0}(z) \\
& +\sum_{j=1}^{k} T_{j}\left(z, C_{1}, C_{2}, \ldots, C_{j}\right) .
\end{aligned}
$$

The expression for the residual is

$$
\begin{aligned}
\mathfrak{R}\left(z, C_{1}, C_{2}, \ldots, C_{k}\right)= & \mathfrak{Q}\left(\widetilde{T}\left(z, C_{1}, C_{2}, \ldots, C_{k}\right)\right) \\
& +f(z) \\
& +\mathfrak{N}\left(\widetilde{T}\left(z, C_{1}, C_{2}, \ldots, C_{k}\right)\right) .
\end{aligned}
$$


If $\Re=0$, then we say that $\widetilde{T}$ is the exact solution, but, in case of nonlinearity, it does not happen generally. To search the constants $C_{k}$, different methods can be applied. One of these methods is the method of least square as follows:

$$
I=\int_{x_{0}}^{x_{1}} \mathfrak{R}^{2}\left(z, C_{1}, C_{2}, \ldots, C_{k}\right) d z .
$$

Minimizing this function, we have

$$
\frac{\partial I}{\partial C_{i}}\left(z, C_{1}, C_{2}, \ldots, C_{k}\right)=0, \quad i=1,2,3, \ldots, k
$$

$x_{0}$ and $x_{1}$ are within the domain of the problem for locating suitable $C_{r}^{\prime} s(r=1,2, \ldots, k)$. Now we solve (11) with boundary conditions (12); we find the following different order problems:

Zeroth-order problem:

$$
\begin{gathered}
T_{0}^{(\mathrm{iv})}(z)=0, \\
T_{0}(0)=0, \\
T_{0}^{\prime \prime}(0)=0, \\
T_{0}(1)=1, \\
T_{0}^{\prime}(1)=T_{0}^{\prime \prime}(1) .
\end{gathered}
$$

First-order problem:

$$
\begin{aligned}
T_{1}^{(\mathrm{iv})}(z)= & C_{1} T_{0}(z) T_{0}^{\prime \prime \prime}(z)+T_{0}^{(\mathrm{iv})}(z)+C_{1} T_{0}^{(\mathrm{iv})}(z) \\
& -C_{1} T_{0}^{\prime \prime}(z) \\
T_{1}(0)= & 0 \\
T_{1}^{\prime \prime}(0)= & 0 \\
T_{1}(1)= & 0 \\
T_{1}^{\prime}(1)= & T_{1}^{\prime \prime}(1) .
\end{aligned}
$$

Second-order problem:

$$
\begin{aligned}
T_{2}^{(\mathrm{iv})}(z)= & C_{2} T_{0}(z) T_{0}^{\prime \prime \prime}(z)+C_{1} T_{1}(z) T_{0}^{\prime \prime \prime}(z) \\
& +C_{1} T_{0}(z) T_{1}^{\prime \prime \prime}(z)+C_{2} T_{0}^{(\mathrm{iv})}+T_{1}^{(\mathrm{iv})}(z) \\
& +C_{1} T_{1}^{(\mathrm{iv})}(z)-C_{2} T_{0}^{\prime \prime}(z)-C_{1} T_{1}^{\prime \prime}(z) \\
T_{2}(0)= & 0 \\
T_{2}^{\prime \prime}(0)= & 0 \\
T_{2}(1)= & 0 \\
T_{2}^{\prime}(1)= & T_{2}^{\prime \prime}(1) .
\end{aligned}
$$

Third-order problem:

$$
\begin{aligned}
T_{3}^{(\mathrm{iv})}(z)= & C_{2} T_{1}(z) T_{0}^{\prime \prime \prime}(z)+C_{1} T_{2}(z) T_{0}^{\prime \prime \prime}(z) \\
& +C_{2} T_{0}(z) T_{1}^{\prime \prime \prime}(z)+C_{1} T_{1}(z) T_{1}^{\prime \prime \prime}(z) \\
& +C_{1} T_{0}(z) T_{2}^{\prime \prime \prime}(z)+C_{2} T_{1}^{(\mathrm{iv})}(z) \\
& +T_{2}^{(\mathrm{iv})}(z)+C_{1} T_{2}^{(\mathrm{iv})}(z)-C_{2} T_{1}^{\prime \prime}(z) \\
& +C_{1} T_{2}^{\prime \prime}(z) \\
T_{3}(0)= & 0 \\
T_{3}^{\prime \prime}(0)= & 0 \\
T_{3}(1)= & 0 \\
T_{3}^{\prime}(1)= & T_{3}^{\prime \prime}(1) .
\end{aligned}
$$

Similarly fourth-order problem can also be found easily. By considering the fourth-order solution, we have

$$
\widetilde{T}(z)=\sum_{i=0}^{4} T_{i}\left(z, C_{1}, C_{2}\right) .
$$

The residual of the problem is

$$
\mathfrak{R e g}=\widetilde{T}^{\text {(iv) }}(z)-\mathscr{M}^{2} \widetilde{T}^{\prime \prime}(z)+\mathscr{R} \widetilde{T}(z) \widetilde{T}^{\prime \prime \prime}(z) .
$$

In order to find $C_{1}$ and $C_{2}$, we apply the method of least square as follows:

$$
\begin{gathered}
J\left(C_{1}, C_{2}\right)=\int_{0}^{1} \operatorname{Reg}^{2}\left(z, C_{1}, C_{2}\right) d z, \\
\frac{\partial J}{\partial C_{i}}\left(z, C_{1}, C_{2}\right)=0, \quad i=1,2 .
\end{gathered}
$$

Solving (75) for $C_{1}, C_{2}$, we get

$$
\begin{aligned}
& C_{1}=-0.89521, \\
& C_{2}=-0.00031 .
\end{aligned}
$$

Using these values of $C_{1}, C_{2}$, the approximate solution is given by

$$
\begin{aligned}
T(z)= & 0.754966 z+0.242565 z^{3}+0.0029716 z^{5} \\
& -0.000509457 z^{7}+3.73464 \times 10^{-6} z^{9} \\
& +3.17424 \times 10^{-6} z^{11}-1.10266 \times 10^{-7} z^{13} \\
& -2.19862 \times 10^{-8} z^{15}+8.28889 \times 10^{-9} z^{17} \\
& +1.26544 \times 10^{-10} z^{19}
\end{aligned}
$$

\section{Conclusion}

In the present paper various analytical techniques are used along with one numerical scheme to find the approximate 
TABLE 1: Absolute residuals for numerical and various analytical schemes.

\begin{tabular}{lcccccc}
\hline$z$ & ADM & DJM & DTM & HPM & OHAM & 0.0 \\
\hline 0.0 & 0.0 & 0.0 & 0.0 & 0.0 & NDSolve \\
0.1 & $1.34 \times 10^{-4}$ & $1.07 \times 10^{-10}$ & $1.40 \times 10^{-17}$ & $8.84 \times 10^{-6}$ & $7.53 \times 10^{-9}$ & $1.20 \times 10^{-2}$ \\
0.2 & $1.06 \times 10^{-3}$ & $1.40 \times 10^{-8}$ & $7.00 \times 10^{-14}$ & $1.76 \times 10^{-5}$ & $6.05 \times 10^{-9}$ & $1.55 \times 10^{-3}$ \\
0.3 & $3.58 \times 10^{-3}$ & $2.50 \times 10^{-7}$ & $1.32 \times 10^{-11}$ & $2.63 \times 10^{-5}$ & $1.66 \times 10^{-8}$ & $2.00 \times 10^{-5}$ \\
0.4 & $8.40 \times 10^{-3}$ & $2.00 \times 10^{-6}$ & $5.61 \times 10^{-10}$ & $3.54 \times 10^{-5}$ & $6.85 \times 10^{-8}$ & $8.02 \times 10^{-6}$ \\
0.5 & $1.62 \times 10^{-2}$ & $1.02 \times 10^{-5}$ & $1.04 \times 10^{-8}$ & $4.56 \times 10^{-5}$ & $1.30 \times 10^{-7}$ & $2.90 \times 10^{-5}$ \\
0.6 & $2.74 \times 10^{-2}$ & $4.10 \times 10^{-5}$ & $1.14 \times 10^{-7}$ & $6.00 \times 10^{-5}$ & $1.24 \times 10^{-7}$ & $6.20 \times 10^{-5}$ \\
0.7 & $4.21 \times 10^{-2}$ & $1.40 \times 10^{-4}$ & $8.63 \times 10^{-7}$ & $7.10 \times 10^{-5}$ & $7.81 \times 10^{-8}$ \\
0.8 & $5.99 \times 10^{-2}$ & $4.00 \times 10^{-4}$ & $5.03 \times 10^{-6}$ & $7.10 \times 10^{-5}$ & $5.34 \times 10^{-7}$ & $1.60 \times 10^{-5}$ \\
0.9 & $7.96 \times 10^{-2}$ & $1.05 \times 10^{-3}$ & $2.40 \times 10^{-5}$ & $9.00 \times 10^{-6}$ & $8.58 \times 10^{-7}$ & $2.90 \times 10^{-4}$ \\
1.0 & $9.89 \times 10^{-2}$ & $2.60 \times 10^{-3}$ & $9.74 \times 10^{-5}$ & $2.60 \times 10^{-4}$ & $4.11 \times 10^{-7}$ & $1.10 \times 10^{-3}$ \\
\hline
\end{tabular}

solution for axisymmetric squeezing flow of incompressible Newtonian fluid having MHD effect and passing through porous medium channel with slip boundary. Absolute residuals of the modeled problem are obtained using these schemes, that is, ADM, DJM, DTM, HPM, and OHAM. Numerical solution is obtained using Mathematica solver NDSolve. The residuals are given in Table 1 which shows the efficiency of all the schemes used in the given scenario as compared with the numerical scheme NDSolve. In comparison with other techniques it is clear from Table 1 that OHAM is more efficient and consistent.

\section{Conflict of Interests}

The authors declare that there is no conflict of interests regarding the publication of this paper.

\section{References}

[1] M. J. Stefan, "Versuch uber die scheinbare adhesion," Akademie der Wissenschaften in Wien. Mathematik-Naturwissen, vol. 69, p. 713,1874 .

[2] X. J. Ran, Q. Y. Zhu, and Y. Li, "An explicit series solution of the squeezing ow between two infinite parallel plates," Communications in Nonlinear Science and Numerical Simulation, vol. 14, pp. 119-132, 2007.

[3] R. J. Grimm, "Squeezing flows of Newtonian liquid films an analysis including fluid inertia," Applied Scientific Research, vol. 32, no. 2, pp. 149-166, 1976.

[4] W. F. Stefa Hughes and R. A. Elco, "Magnetohydrodynamic lubrication flow between parallel rotating disks," Journal of Fluid Mechanics, vol. 13, pp. 21-32, 1962.

[5] S. Kamiyama, "Inertia effects in MHD hydrostatic thrust bearing," Journal of Lubrication Technology, vol. 91, no. 4, pp. 589596, 1969.

[6] E. A. Hamza, “The magnetohydrodynamic squeeze film," Journal of Tribology, vol. 110, no. 2, pp. 375-377, 1988.

[7] S. Bhattacharyya and A. Pal, "Unsteady MHD squeezing flow between two parallel rotating discs," Mechanics Research Communications, vol. 24, no. 6, pp. 615-623, 1997.

[8] W. F. Hughes and R. A. Elco, "Magnetohydrodynamic lubrication flow between parallel rotating disks," Journal of Fluid Mechanics, vol. 13, no. 1, pp. 21-32, 1962.
[9] I. Ullah, M. T. Rahim, and H. Khan, "Application of Daftardar Jafari method to first grade MHD squeezing fluid flow in a porous medium with slip boundary condition," Abstract and Applied Analysis, vol. 2014, Article ID 479136, 8 pages, 2014.

[10] J.-H. He, "Homotopy perturbation method for solving boundary value problems," Physics Letters A, vol. 350, no. 1-2, pp. 87$88,2006$.

[11] J.-H. He, "Application of homotopy perturbation method to nonlinear wave equations," Chaos, Solitons and Fractals, vol. 26, no. 3, pp. 695-700, 2005.

[12] V. Marinca, N. Herişanu, and I. Nemeş, "Optimal homotopy asymptotic method with application to thin film flow," Central European Journal of Physics, vol. 6, no. 3, pp. 648-653, 2008.

[13] V. Marinca and N. Herisanu, "An optimal homotopy asymptotic method for solving nonlinear equations arising in heat transfer," International Communications in Heat and Mass Transfer, vol. 35, pp. 710-715, 2008.

[14] N. Herisanu, V. Marinca, and G. Madescu, "An analytical approach to non-linear dynamical model of a permanent magnet synchronous generator," Wind Energy, vol. 18, pp. 1657-1670, 2015.

[15] J. K. Zhou, Deferential Transformation and Its Application for Electrical Circuits, Huazhong University Press, Wuhan, China, 1986.

[16] S. Islam, H. Khan, I. A. Shah, and G. Zaman, "An axisymmetric squeezing fluid flow between the two infinite parallel plates in a porous medium channel," Mathematical Problems in Engineering, vol. 2011, Article ID 349803, 10 pages, 2011.

[17] I. Ullah, M. T. Rahim, and H. Khan, "Approximation of first grade MHD squeezing fluid flow with slip boundary condition using DTM and OHAM," Mathematical Problems in Engineering, vol. 2013, Article ID 816262, 9 pages, 2013.

[18] F. Ayaz, "On the two-dimensional differential transform method," Applied Mathematics and Computation, vol. 143, no. 2-3, pp. 361-374, 2003.

[19] I. H. A.-H. Hassan, "Comparison differential transformation technique with Adomian decomposition method for linear and nonlinear initial value problems," Chaos, Solitons \& Fractals, vol. 36, no. 1, pp. 53-65, 2008.

[20] G. Adomian, Nonlinear Stochastic Systems: Theory and Applications to Physics, vol. 46 of Mathematics and its Applications, Kluwer Academic Press, Dordrecht, The Netherlands, 1989.

[21] G. Adomian, Solving Frontier Problems of Physics: The Decomposition Method, Kluwer Academic, 1994. 
[22] A.-M. Wazwaz, "The numerical solution of sixth-order boundary value problems by the modified decomposition method," Applied Mathematics and Computation, vol. 118, no. 2-3, pp. 311325,2001

[23] V. Daftardar-Gejji and H. Jafari, "An iterative method for solving nonlinear functional equations," Journal of Mathematical Analysis and Applications, vol. 316, no. 2, pp. 753-763, 2006.

[24] V. Daftardar-Gejji and S. Bhalekar, "Solving fractional boundary value problems with Dirichlet boundary conditions using a new iterative method," Computers \& Mathematics with Applications, vol. 59, no. 5, pp. 1801-1809, 2010.

[25] I. Ullah, H. Khan, and M. T. Rahim, "Numerical solutions of fifth and sixth order nonlinear boundary value problems by Daftardar Jafari method," Journal of Computational Engineering, vol. 2014, Article ID 286039, 8 pages, 2014.

[26] N. B. Naduvinamani, P. S. Hiremath, and G. Gurubasavaraj, "Static and dynamic behaviour of squeeze-film lubrication of narrow porous journal bearings with coupled stress fluid," Proceedings of the Institution of Mechanical Engineers Part J: Journal of Engineering Tribology, vol. 215, no. 1, pp. 45-62, 2001.

[27] W.-P. Breugem, "The effective viscosity of a channel-type porous medium," Physics of Fluids, vol. 19, no. 10, Article ID 103104, 2007.

[28] H. Liu and Y. Song, "Differential transform method applied to high index differential-algebraic equations," Applied Mathematics and Computation, vol. 184, no. 2, pp. 748-753, 2007.

[29] S.-H. Chang and I.-L. Chang, "A new algorithm for calculating one-dimensional differential transform of nonlinear functions," Applied Mathematics and Computation, vol. 195, no. 2, pp. 799$808,2008$. 

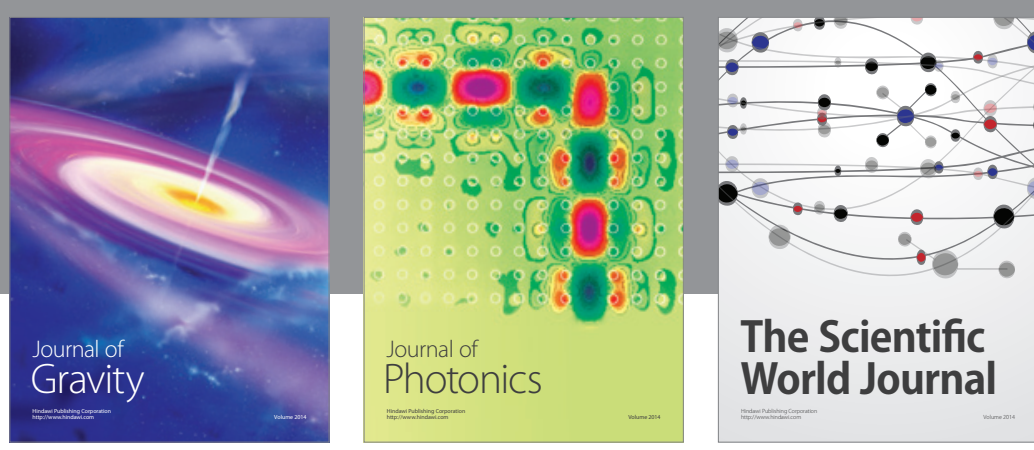

The Scientific World Journal
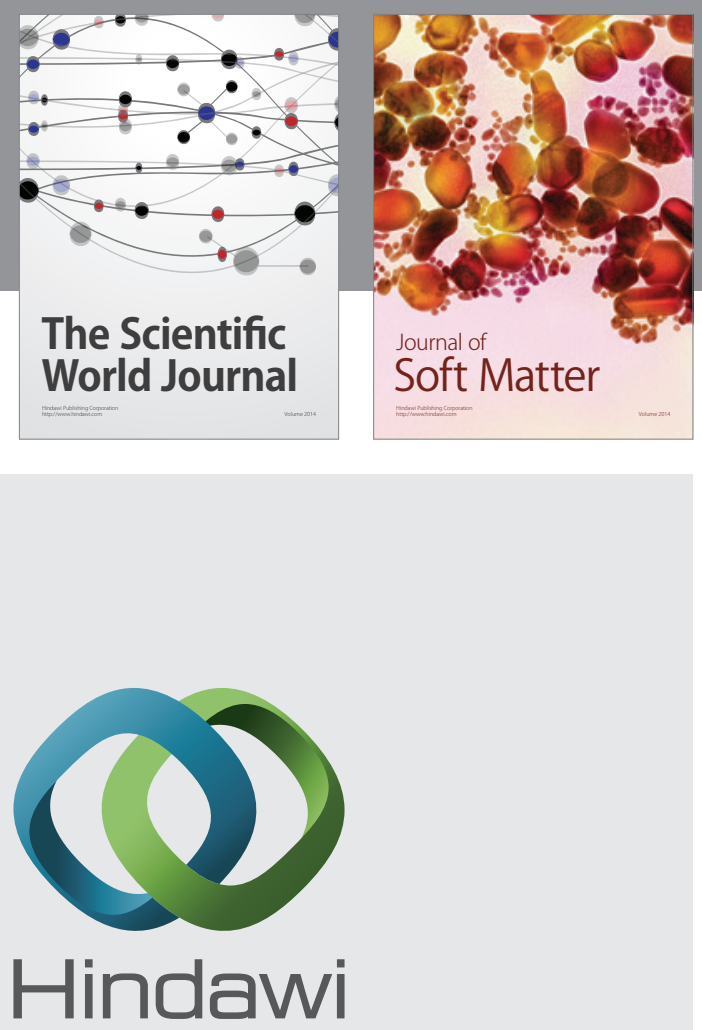

Submit your manuscripts at

http://www.hindawi.com

nternational Journal of

Statistical Mechanics
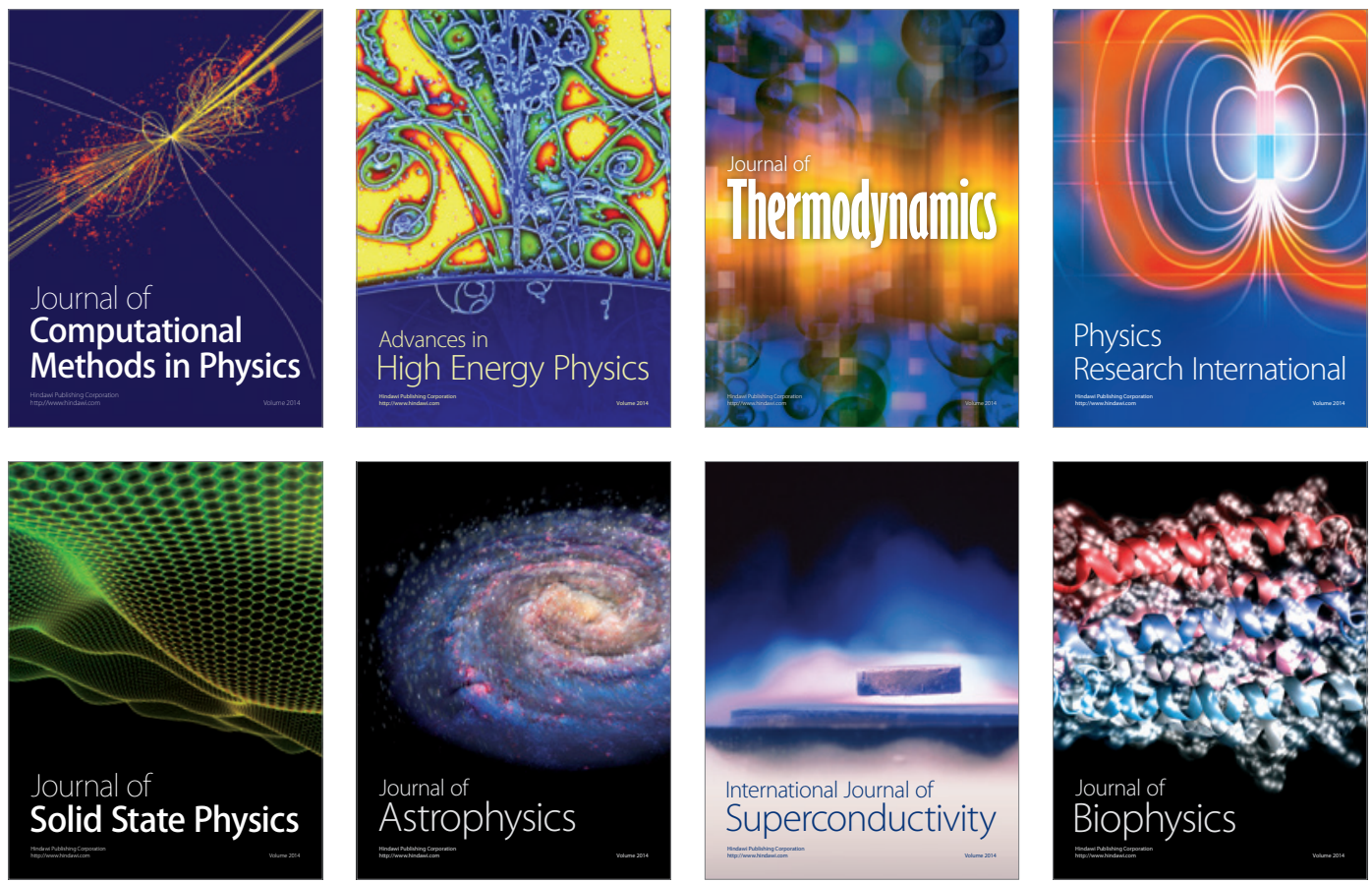
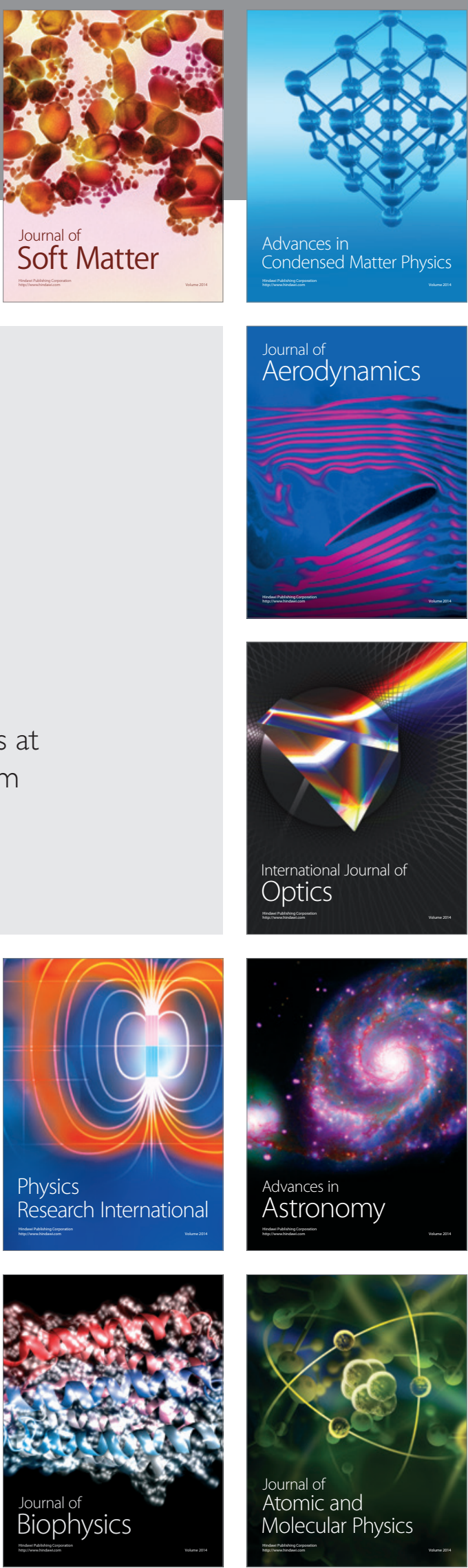